\title{
Association between tooth loss and cognitive impairment in community- dwelling older Japanese adults: a 4-year prospective cohort study from the Ohasama study
}

Sho Saito ${ }^{1}$, Takashi Ohi ${ }^{1,2^{*}}$ (D), Takahisa Murakami ${ }^{3}$, Takamasa Komiyama', Yoshitada Miyoshi ${ }^{1}$, Kosei Endo ${ }^{1}$, Michihiro Satoh ${ }^{3}$, Kei Asayama ${ }^{4,5}$, Ryusuke Inoue ${ }^{6}$, Masahiro Kikuya ${ }^{7}$, Hirohito Metoki ${ }^{3}$, Yutaka Imai ${ }^{5}$,

Takayoshi Ohkubo ${ }^{4}$ and Yoshinori Hattori ${ }^{1}$

\begin{abstract}
Background: Numerous prospective studies have investigated the association between the number of remaining teeth and dementia or cognitive decline. However, no agreement has emerged on the association between tooth loss and cognitive impairment, possibly due to past studies differing in target groups and methodologies. We aimed to investigate the association between tooth loss, as evaluated through clinical oral examinations, and the development of cognitive impairment in community-dwelling older adults while considering baseline cognitive function.

Methods: This 4-year prospective cohort study followed 140 older adults (69.3\% female) without cognitive impairment aged $\geq 65$ years (mean age: $70.9 \pm 4.3$ years) living in the town of Ohasama, Iwate Prefecture, Japan. Cognitive function was evaluated with the Mini-Mental State Examination (MMSE) in baseline and follow-up surveys. Based on a baseline oral examination, the participants were divided into those with 0-9 teeth and those with $\geq 10$ teeth. To investigate the association between tooth loss and cognitive impairment, we applied a multiple logistic regression analysis adjusted for age, sex, hypertension, diabetes, cerebrovascular/cardiovascular disease, hypercholesterolemia, depressive symptoms, body mass index, smoking status, drinking status, duration of education, and baseline MMSE score.
\end{abstract}

Results: In the 4 years after the baseline survey, 27 participants (19.3\%) developed cognitive impairment (i.e., MMSE scores of $\leq 24$ ). Multiple logistic regression analysis indicated that participants with $0-9$ teeth were more likely to develop cognitive impairment than those with $\geq 10$ teeth were (odds ratio: 3.31; 95\% confidence interval: 1.07-10.2). Age, male gender, and baseline MMSE scores were also significantly associated with cognitive impairment.

Conclusions: Tooth loss was independently associated with the development of cognitive impairment within 4 years among community-dwelling older adults. This finding corroborates the hypothesis that tooth loss may be a predictor or risk factor for cognitive decline.

Keywords: Cognitive impairment, Cohort study, Community-dwelling, Elderly, Tooth loss

\footnotetext{
* Correspondence: tohi@dent.tohoku.ac.jp

'Division of Aging and Geriatric Dentistry, Department of Oral Function and

Morphology, Tohoku University Graduate School of Dentistry, 4-1

Seiryo-machi, Aoba-ku, Sendai, Miyagi 980-8575, Japan

2Japanese Red Cross Ishinomaki Hospital, Ishinomaki, Japan

Full list of author information is available at the end of the article
}

(c) The Author(s). 2018 Open Access This article is distributed under the terms of the Creative Commons Attribution 4.0 International License (http://creativecommons.org/licenses/by/4.0/), which permits unrestricted use, distribution, and reproduction in any medium, provided you give appropriate credit to the original author(s) and the source, provide a link to the Creative Commons license, and indicate if changes were made. The Creative Commons Public Domain Dedication waiver (http://creativecommons.org/publicdomain/zero/1.0/) applies to the data made available in this article, unless otherwise stated. 


\section{Background}

Dementia is the leading reason for older people needing long-term care in Japan. It is also a major social problem because the need for long-term care not only reduces the patient's quality of life but also places psychological and financial burdens on the family and other caregivers. According to World Health Organization estimates, the number of people with dementia worldwide reached around 50 million in 2017 and is increasing by nearly 10 million new cases annually [1]. Because no effective treatment for dementia has been established, the modifiable predictors and risk factors must be identified in order to reduce the incidence of dementia.

Numerous prospective studies [2-13] have reported an association between oral health, particularly the number of remaining teeth, and dementia or cognitive decline in old age. Conversely, some reports [14-18] found no such association. Recent systematic reviews [19-21] have reached no consensus position on the question. The inconsistencies between studies may arise from differences in target groups and methodologies. Some studies have analyzed specific occupational groups or nursing home residents $[3-5,9,12,14]$. Several studies have determined the number of remaining teeth through subject-administered questionnaires $[7-10,15,18]$, whereas some others have used clinical oral examinations $[2,11,13,16,17]$. Furthermore, although baseline cognitive function is closely related to subsequent cognitive decline, only two studies $[8,11]$ have examined baseline cognitive function as a confounding factor.

We therefore aimed to elucidate the association between tooth loss, as evaluated through oral examinations, and the subsequent development of cognitive impairment in community-dwelling older adults while considering baseline cognitive function.

\section{Methods}

\section{Study design and population}

This study was performed as a component of the Ohasama Study, a prospective cohort study on hypertension/cardiovascular disease that has followed general inhabitants of Ohasama, a rural town in northern Japan's Iwate Prefecture, since 1986 [22, 23]. Figure 1 shows the study flow diagram. From 2005 to 2012, 448 inhabitants aged $\geq 65$ years participated in the baseline survey. Of them, we excluded 40 participants with missing data and 82 participants who exhibited cognitive decline on cognitive function tests. The remaining 326 inhabitants were included as participants. Of them, 150 (46\%) participated in the follow-up survey between 2009 and 2016. After excluding 10 participants with incomplete follow-up survey data, we included 140 participants in the final analysis as the "follow-up group." The mean ( \pm standard deviation) follow-up period was $4.0 \pm$
0.1 years. We included 176 people who did not participate in the follow-up survey in our "drop-out group."

This study was approved by the Institutional Review Board of the Tohoku University Graduate School of Pharmaceutical Science and conformed to the principles of the Declaration of Helsinki. Written informed consent to participate was obtained from all participants.

\section{Measurements}

Tooth loss at the time of the baseline survey was evaluated via count of the number of remaining teeth by specially trained dentists. The median number of remaining teeth was 10 , so the participants were divided into those with $0-9$ teeth and those with $\geq 10$ teeth. We defined having 0-9 teeth as "multiple tooth loss."

Cognitive function was evaluated with the Mini-Mental State Examination (MMSE) [24] in the baseline and follow-up surveys. Participants with a total score of $\leq 24$ (full score: 30 points) were defined as having cognitive impairment. This cut-off reportedly achieves $83 \%$ sensitivity and $93 \%$ specificity in diagnosing dementia in Japanese patients [25]. We defined hypertension as a home blood pressure of $\geq 135 / 85 \mathrm{mmHg}$ [26], use of antihypertensive medications, or a history of hypertension; diabetes mellitus as a non-fasting glucose concentration of $\geq 200 \mathrm{mg} / \mathrm{dL}$ [27], a glycated hemoglobin concentration of $\geq 6.5 \%$, use of anti-diabetes medications, or a history of diabetes mellitus; cerebrovascular/cardiovascular disease as a history of atrial fibrillation, heart disease, or cerebrovascular disease; and hypercholesterolemia as a total cholesterol level of $\geq 220 \mathrm{mg} / \mathrm{dL}$, use of anti-hypercholesterolemia medications, or a history of hypercholesterolemia. Depressive symptoms were evaluated with the Zung Self-Rating Depression Scale (SDS) [28]. The maximum SDS score is 80 , and participants with scores of $\geq 40$ were defined as having depressive symptoms. Body mass index (BMI) was calculated as weight in kilograms divided by height in meters squared. Participants were categorized as being current smokers/drinkers or not being current smokers/drinkers. Duration of education was categorized as being either less than 10 years or at least 10 years.

\section{Statistical analysis}

Bivariate analyses were performed with Student's $t$-test or the Wilcoxon rank sum test for continuous variables and the chi-squared test for categorical variables. A multiple logistic regression analysis was used to calculate adjusted odds ratios (ORs) and their 95\% confidence intervals (CIs) for the development of cognitive impairment within 4 years of the baseline survey. Because few participants developed cognitive impairment, we applied logistic regression with penalized maximum likelihood 


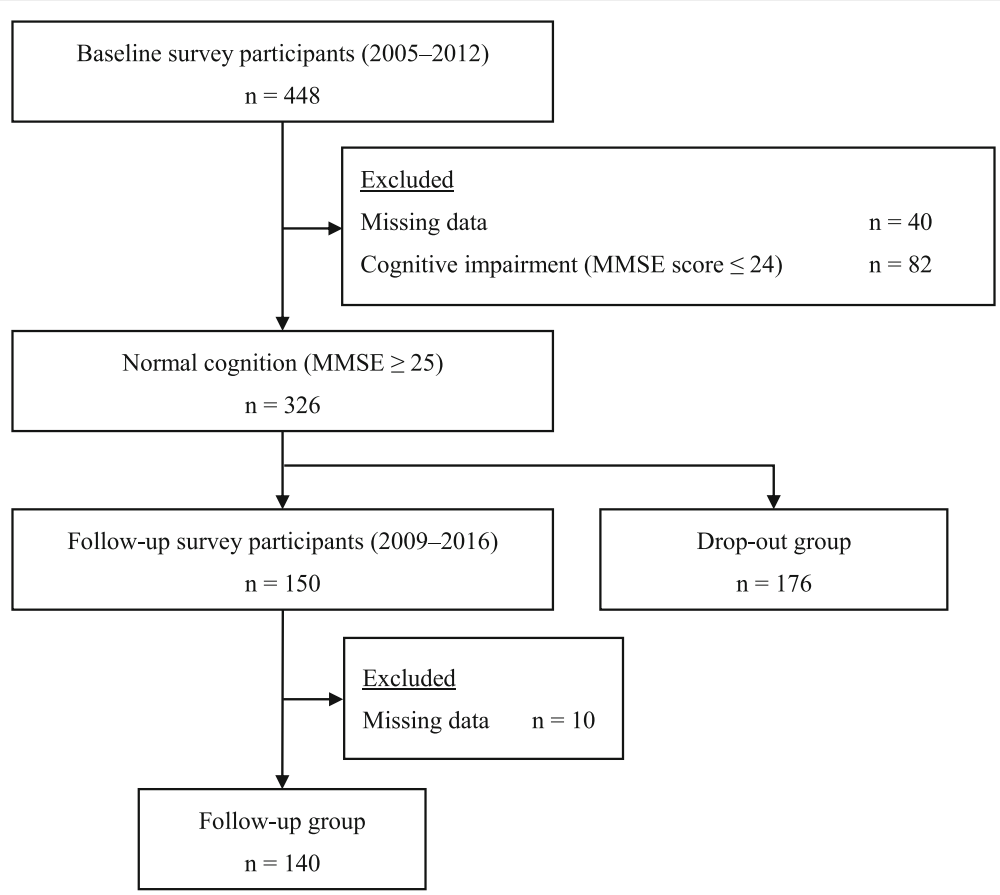

Fig. 1 Flow diagram of study participants

estimation for rare events analysis [29]. The statistical significance threshold was $p<0.05$ for all tests. Statistical analyses were performed with JMP Pro 12 (SAS Institute, Cary, NC, USA) and Stata 14 (StataCorp LLC, College Station, TX, USA).

\section{Results}

The baseline characteristics of the follow-up and drop-out groups are shown in Table 1 . The drop-out participants were significantly older and more likely to have hypertension than the follow-up group participants were, but the two groups did not significantly differ in sex, duration of education, BMIs, baseline MMSE scores, or frequencies of multiple tooth loss, current smoker status, current drinker status, diabetes mellitus, cerebrovascular/cardiovascular disease, hypercholesterolemia, or depressive symptoms. Of the follow-up participants, 27 (19.3\%) had cognitive impairment at the follow-up

Table 1 Characteristics of the follow-up and drop-out groups

\begin{tabular}{llll}
\hline & Follow-up $(n=140)$ & Drop-out $(n=176)$ & $P$-value \\
\hline Age (mean \pm SD) & $70.9 \pm 4.3$ & $73.2 \pm 4.8$ & $<0.0001$ \\
Male (\%) & 30.7 & 29.0 & 0.7 \\
Hypertension (\%) & 53.6 & 66.5 & 0.02 \\
Diabetes (\%) & 11.4 & 17.6 & 0.1 \\
Cerebrovascular/cardiovascular disease (\%) & 12.9 & 15.3 & 0.5 \\
Hypercholesterolemia (\%) & 60.7 & 54.0 & 0.2 \\
Depressive symptoms (\%) & 10.7 & 10.2 & 0.9 \\
BMI (mean \pm SD) & $23.6 \pm 3.2$ & $23.9 \pm 2.8$ & 0.4 \\
Current smoker (\%) & 7.1 & 5.7 & 0.6 \\
Current drinker (\%) & 39.3 & 32.4 & 0.2 \\
$<10$ years of education (\%) & 73.6 & 76.7 & 0.5 \\
Baseline MMSE score (mean \pm SD) & $27.7 \pm 1.6$ & $27.8 \pm 1.5$ & 0.4 \\
Multiple tooth loss (\%) & 50.7 & 46.6 & 0.5 \\
\hline P-valus were der & \\
\hline
\end{tabular}

$P$-values were determined with Student's $t$-test or the Wilcoxon rank sum test for continuous variables and the chi-squared test for categorical variables. Multiple tooth loss was defined as having 0-9 remaining teeth 
survey. Table 2 compares the baseline characteristics of individuals who retained normal cognition and those who developed cognitive impairment by the follow-up survey. Compared to the participants with normal cognition, those with cognitive impairment were significantly older, had significantly lower baseline MMSE scores, and were significantly more likely to be male and to exhibit multiple tooth loss.

Table 3 shows the ORs and 95\% CIs for developing cognitive impairment. In the age- and sex-adjusted model, multiple tooth loss was associated with an increased likelihood of developing cognitive impairment (OR: 3.39; 95\% CI: 1.29-8.88). This association remained (OR: 3.31; 95\% CI: 1.07-10.2) even after adjusting for age, sex, hypertension, diabetes, cerebrovascular/cardiovascular disease, hypercholesterolemia, depressive symptoms, BMI, smoking status, drinking status, duration of education, and baseline MMSE score. Greater ages, male gender, and lower baseline MMSE scores were also significantly associated with developing cognitive impairment. We did not find an interaction between age and tooth loss $(p=0.925)$.

\section{Discussion}

In the present study, we examined whether multiple tooth loss, as evaluated with professional clinical oral examinations, is associated with developing cognitive impairment in community-dwelling older adults. We found that the presence of multiple tooth loss significantly increased the risk of developing cognitive impairment within 4 years independently of age, sex, hypertension, diabetes, cerebrovascular/cardiovascular disease, hypercholesterolemia, depressive symptoms, BMI, smoking status, drinking status, duration of education, and baseline MMSE score. This finding suggests that maintaining healthy dentition reduces the risk of cognitive impairment.

This study is one of the few longitudinal studies $[2,11,13,16,17]$ to investigate the association between tooth loss, as evaluated with oral examinations, and cognitive impairment in community-dwelling older adults. A survey of community-dwelling older adults in South Korea [2] reported that a paucity of remaining teeth was related to the onset of dementia within 2.4 years. A 5-year prospective cohort study of Japanese community residents [11] reported that tooth loss predicted the development of mild memory impairment, and another 5-year cohort study of older adults in Japan [13] found that tooth loss was associated with an increased risk of developing all-cause dementia and Alzheimer's disease. Our observation of a significant association between tooth loss and cognitive dysfunction corroborates these previous studies' results. However, some studies $[16,17]$ found no association between tooth loss and cognitive decline. These studies differ from the others in that the participants were all older women [17] or in that the participants included middle-aged people as well as older ones [16].

In this study, we included the baseline MMSE score as a confounding factor in the multivariate analysis in order to consider the possible effect of mild cognitive decline that went undetected in screening tests. However, multiple tooth loss remained associated with cognitive impairment even after adjusting for baseline MMSE scores. Only two previous studies $[8,11]$ have adjusted for baseline cognitive function. A cohort study of older Japanese adults [8] found that having few teeth without

Table 2 Baseline characteristics of individuals with normal cognition and those who developed cognitive impairment

\begin{tabular}{llll}
\hline & Normal cognition $(n=113)$ & Cognitive impairment $(n=27)$ & $P$-value \\
\hline Age (mean \pm SD) & $70.5 \pm 4.2$ & $72.3 \pm 4.5$ & 0.04 \\
Male (\%) & 26.6 & 48.2 & 0.03 \\
Hypertension (\%) & 52.2 & 59.3 & 0.5 \\
Diabetes (\%) & 9.7 & 18.5 & 0.2 \\
Cerebrovascular/cardiovascular disease (\%) & 11.5 & 18.5 & 0.3 \\
Hypercholesterolemia (\%) & 61.1 & 59.3 & 0.9 \\
Depressive symptoms (\%) & 8.9 & 18.5 & 0.1 \\
BMl (mean \pm SD) & $23.4 \pm 3.0$ & $24.3 \pm 4.0$ & 0.2 \\
Current smoker (\%) & 7.1 & 7.4 & 0.9 \\
Current drinker (\%) & 41.6 & 29.6 & 0.3 \\
$<10$ years of education (\%) & 71.7 & 81.5 & 0.3 \\
Baseline MMSE score (mean \pm SD) & $28.0 \pm 1.6$ & $26.4 \pm 1.2$ & $<0.0001$ \\
Multiple tooth loss (\%) & 46.0 & 70.4 & 0.02 \\
\hline Pvalues we & \\
\hline
\end{tabular}

$P$-values were determined with Student's $t$-test or the Wilcoxon rank sum test for continuous variables and the chi-squared test for categorical variables. Multiple tooth loss was defined as having 0-9 remaining teeth

$B M I$ body mass index, MMSE Mini-Mental State Examination, SD standard deviation 
Table 3 Multiple logistic regression model for development of cognitive impairment

\begin{tabular}{|c|c|c|c|c|}
\hline & \multicolumn{2}{|c|}{ Age- and sex-adjusted model } & \multicolumn{2}{|c|}{ Fully adjusted model $^{a}$} \\
\hline & OR $(95 \% \mathrm{Cl})$ & $P$-value & OR $(95 \% \mathrm{Cl})$ & $P$-value \\
\hline Age & $1.07(0.98-1.18)$ & 0.1 & $1.14(1.01-1.29)$ & 0.03 \\
\hline Male gender & $3.34(1.32-8.46)$ & 0.01 & $4.60(1.25-16.7)$ & 0.02 \\
\hline Hypertension & & & $1.28(0.44-3.70)$ & 0.6 \\
\hline Diabetes & & & $3.77(0.79-18.0)$ & 0.1 \\
\hline Cerebrovascular/cardiovascular disease & & & $2.62(0.68-10.1)$ & 0.2 \\
\hline Hypercholesterolemia & & & $1.68(0.56-5.00)$ & 0.3 \\
\hline Depressive symptoms & & & $2.50(0.54-11.4)$ & 0.2 \\
\hline BMI & & & $1.04(0.87-1.25)$ & 0.6 \\
\hline Current smoker & & & $0.48(0.69-3.42)$ & 0.5 \\
\hline Current drinker & & & $0.50(0.14-1.72)$ & 0.3 \\
\hline Duration of education & & & $1.31(0.33-5.14)$ & 0.7 \\
\hline Baseline MMSE score & & & $0.48(0.31-0.74)$ & 0.001 \\
\hline Multiple tooth loss & $3.39(1.29-8.88)$ & 0.013 & $3.31(1.07-10.2)$ & 0.037 \\
\hline
\end{tabular}

The objective variable for the multiple logistic regression analysis was whether cognitive function declined within 4 years, and the explanatory variable was whether multiple tooth loss was present. Multiple tooth loss was defined as having $0-9$ remaining teeth

${ }^{a}$ Adjusted for age, sex, hypertension, diabetes, cerebrovascular/cardiovascular disease, hypercholesterolemia, depressive symptoms, BMI, current smoker status, current drinker status, duration of education, and baseline MMSE score

$B M I$ body mass index, $\mathrm{Cl}$ confidence interval, MMSE Mini-Mental State Examination, $O R$ odds ratio, SD standard deviation

dentures was associated with an increased risk of dementia independently of forgetfulness. A 5-year prospective cohort study of Japanese community residents [11] found that tooth loss was associated with developing mild memory impairment even after adjusting for baseline MMSE scores.

Several mechanisms may explain the association between tooth loss and cognitive impairment. First, the association may depend on chronic inflammation arising from periodontal disease, which is a major cause of tooth loss in later life. Previous studies showed that periodontal disease was the most common reason for tooth extraction in patients older than 45 years $[30,31]$. Therefore, chronic exposure to periodontal diseaserelated inflammation is presumably a more serious problem in older adults with multiple tooth loss than in those without multiple tooth loss. Clinical attachment loss [32] and alveolar bone resorption [33], both of which indicate the cumulative history of periodontal disease, are associated with cognitive impairment. Systemic inflammatory reactions arising from periodontal disease are a hypothesized risk factor for Alzheimer's disease [34-36].

Second, the association may depend on changes in food intake and nutritional status due to tooth loss. Poor nutritional status and nutrient deficiencies are reportedly associated with cognitive decline and Alzheimer's disease [37-39]. A nationwide survey in the United States [40] found that individuals with fewer than 28 teeth had significantly lower intakes of carrots, tossed salads, and dietary fiber and had lower serum concentrations of $\beta$-carotene, folic acid, and vitamin $\mathrm{C}$ relative to individuals who were fully dentate. A survey of older Japanese adults [41] found that tooth loss was associated with decreased intakes of vegetables, fish, and shellfish. Therefore, macronutrient and micronutrient deficiencies resulting from decreased masticatory performance may contribute to cognitive impairment in older adults with missing teeth.

Third, the association may arise from deteriorations in masticatory performance reducing brain stimulation. Mastication-related trigeminal nerve sensory inputs reportedly increase cerebral blood flow [42], which in turn promotes arousal and activates a broad region that includes the sensory motor cortex, supplementary motor cortex, insular cortex, thalamus, and cerebellum [43]. Alzheimer's disease model rats fed soft foods exhibit deficiencies in memory and learning abilities relative to rats fed hard foods [44], and rats with extracted molars exhibit reduced acetylcholine release in the cerebral cortex and deficiencies in spatial memory [45]. Therefore, masticatory dysfunction due to tooth loss may adversely affect brain function and thereby promote cognitive impairment.

Besides tooth loss, the factors significantly associated with cognitive impairment were advanced age, male gender, and low baseline MMSE scores. However, unlike tooth loss, these additional factors are unmodifiable. Practicing proper oral health to maintain healthy dentition may reduce the risk of future cognitive impairment. Furthermore, maintaining masticatory performance through prostheses and balanced nutritional intakes may 
reduce the negative impact of tooth loss on cognitive function. A 4-year prospective cohort study of individuals aged $\geq 65$ years in Japan's Aichi Prefecture [8] found that those with few teeth and no dentures had an increased risk of dementia relative to those with $\geq 20$ teeth, but the risk of dementia was not significantly elevated in individuals with few teeth who used dentures. Intervention studies are needed to clarify whether restoring masticatory function and balancing nutritional intakes can prevent cognitive impairment.

This study has several limitations. First, the follow-up rate was not high (46\%), and the follow-up pool might have been biased towards individuals who were healthier and had greater health awareness. Therefore, the possibility of selection bias should be considered when generalizing the present findings. However, this bias will not affect the internal validity of the relationship between tooth loss and the onset of cognitive impairment. Further, the follow-up and drop-out groups did not differ in any baseline characteristics except for age and hypertension rates. As for oral health, the participants' average baseline number of teeth, measured from 2005 to 2012, was approximately five fewer than the average number in age-matched older Japanese adults recorded in national survey data from 2011 [46]. This suggests that our participants might not have been in good oral health. In addition, we may have lacked the participation of elderly people who were developing severe cognitive impairment during the 4 year follow-up; therefore, there is a possibility that the association between tooth loss and cognitive impairment was underestimated. Second, we did not evaluate dementia onset as an outcome. Instead, we screened for dementia with the MMSE, but this test is an indicator of cognitive function used worldwide in research and clinical practice. Its reliability for diagnosing dementia has been shown for Japanese patients [25]. Third, the timecourse of tooth loss was not taken into account. A previous prospective study showed that the rates of tooth loss and periodontal disease progression predicted subsequent cognitive decline [4]. Fourth, there is the problem of reverse causality. A previous study reported that cognitive decline was associated with increased odds of complete tooth loss, infrequent toothbrushing, and higher plaque levels [47]. Decline in cognitive function might increase the risk of tooth loss through poor oral hygiene because of reductions in oral health consciousness. In the present study, participants with cognitive decline at the baseline survey were excluded and the baseline MMSE scores were included as a confounding factor in the multivariate analyses to avoid the effect of reverse causality; however, there is the possibility that some participants who lost their teeth due to the influence of mild cognitive decline that was not detected in our evaluation were included.
Finally, we could not adjust for all possible confounding factors. For example, low socioeconomic status, absence of spouse, unhealthy lifestyles, and certain genes are potentially associated with cognitive decline, but we could not collect data on these factors (although duration of education is a potential proxy for socioeconomic status). However, we did control for many potential confounders, including medical history, based on measurements from medical examinations, and our findings were robust.

\section{Conclusions}

This prospective cohort study of community-dwelling older adults indicated that multiple tooth loss was associated with the development of cognitive impairment. This finding supports the hypothesis that tooth loss is a predictor or risk factor for cognitive decline. Both tooth loss and cognitive impairment are chronically accumulated over time. Further studies should be conducted to confirm the true nature of the impacts of tooth loss on cognitive impairment.

\section{Abbreviations}

BMI: body mass index; Cl: confidence interval; MMSE: Mini-Mental State Examination; OR: odds ratio; SDS: Zung Self-Rating Depression Scale

\begin{abstract}
Acknowledgements
The authors are grateful to Dr. Mikio Hirano (Department of Community Medical Support, Tohoku University, Sendai, Japan) and Dr. Aya Hosokawa (Center for Gerontology and Social Science, National Center for Geriatrics and Gerontology, Aichi, Japan) for advice on measuring MMSE scores and to Dr. Jun Aida (Center for Epidemiology, Biostatistics and Clinical Research, Tohoku University Graduate School of Dentistry, Sendai, Japan) for advice on data analysis and other considerations.
\end{abstract}

\section{Funding}

This study was partly supported by Grants-in-Aid for Scientific Research (C) (15 K11146 and 16 K11850) and Grants-in-Aid for Young Scientists (B) (22792106, 23792202, 25861821, and 25862072) from the Ministry of Education, Culture, Sports, Science and Technology of Japan and by a Health Labour Sciences Research Grant (H26-Junkankitou [Seisaku]-Ippan-001) from the Ministry of Health, Labour and Welfare of Japan. This study's funding sources played no part in the design, methods, participant recruitment, data collection, analysis, or preparation of this paper.

\section{Availability of data and materials \\ The datasets generated and/or analyzed during the current study are not publicly available due the need to protect the participants' anonymity.}

\section{Authors' contributions}

$\mathrm{SS}, \mathrm{TO}, \mathrm{TK}, \mathrm{YI}, \mathrm{TO}$, and $\mathrm{YH}$ generated the study concept and design. SS, TO, $\mathrm{YM}, \mathrm{TK}, \mathrm{KE}, \mathrm{MS}, \mathrm{KA}, \mathrm{RI}, \mathrm{MK}, \mathrm{HM}, \mathrm{YI}, \mathrm{TO}$, and $\mathrm{YH}$ acquired and organized the data. SS, TO, TM, TK, YM, and KE analyzed and interpreted the data. SS, TO, $T M$, TK, and $Y H$ wrote the first draft of the manuscript. SS, TO, TM, TK, YM, KE, $\mathrm{TO}$, and $\mathrm{YH}$ contributed critical revisions and important intellectual content to the manuscript. All authors read and approved the final manuscript.

\section{Ethics approval and consent to participate}

This study was approved by the Institutional Review Board of Tohoku University Graduate School of Pharmaceutical Science (approval number: 10-08) and conformed to the principles of the Declaration of Helsinki. All participants gave written informed consent to participate, and their anonymity was preserved. 


\section{Consent for publication}

Not applicable.

\section{Competing interests}

The authors declare that they have no competing interests.

\section{Publisher's Note}

Springer Nature remains neutral with regard to jurisdictional claims in published maps and institutional affiliations.

\section{Author details}

'Division of Aging and Geriatric Dentistry, Department of Oral Function and Morphology, Tohoku University Graduate School of Dentistry, 4-1

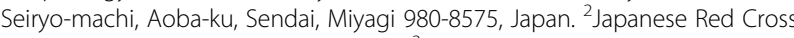
Ishinomaki Hospital, Ishinomaki, Japan. ${ }^{3}$ Division of Public Health, Hygiene and Epidemiology, Faculty of Medicine, Tohoku Medical and Pharmaceutical University, Sendai, Japan. ${ }^{4}$ Department of Hygiene and Public Health, Teikyo University School of Medicine, Tokyo, Japan. ${ }^{5}$ Department of Planning for Drug Development and Clinical Evaluation, Tohoku University Graduate School of Pharmaceutical Sciences, Sendai, Japan. ${ }^{6}$ Department of Medical Informatics, Tohoku University Graduate School of Medicine, Sendai, Japan. ${ }^{7}$ Department of Preventive Medicine and Epidemiology, Tohoku Medical Megabank Organization, Tohoku University, Sendai, Japan.

Received: 9 January 2018 Accepted: 3 August 2018

Published online: 20 August 2018

\section{References}

1. World Health Organization. Dementia fact sheet. 2017. http://www.who.int/ mediacentre/factsheets/fs362/en. Accessed 7 Aug 2018.

2. Kim JM, Stewart R, Prince M, Kim SW, Yang SJ, Shin IS, et al. Dental health, nutritional status and recent-onset dementia in a Korean community population. Int J Geriatr Psychiatry. 2007;22:850-5.

3. Stein PS, Desrosiers M, Donegam SJ, Yepes JF, Kryscio RJ. Tooth loss, dementia and neuropathology in the Nun study. J Am Dent Assoc. 2007:138:1314-22

4. Kaye EK, Valencia A, Baba N, Spiro A III, Dietrich T, Garcia RI. Tooth loss and periodontal disease predict poor cognitive function in older men. J Am Geriatr Soc. 2010;58:713-8.

5. Stein PS, Kryscio RJ, Desrosiers M, Donegan SJ, Gibbs MB. Tooth loss, apolipoprotein E, and decline in delayed word recall. J Dent Res. 2010;89:473-7.

6. Arrive E, Letenneur L, Matharan F, Laporte C, Helmer C, Barberger-Gateau P, et al. Oral health condition of French elderly and risk of dementia: a longitudinal cohort study. Community Dent Oral Epidemiol. 2012;40:230-8

7. Paganini-Hill WSC, Atchison A. Dentition, dental health habits, and dementia: The Leisure World Cohort Study. J Am Geriatr Soc. 2012;60:1556-63.

8. Yamamoto $\mathrm{T}$, Kondo K, Hirai H, Nakade M, Aida J, Hirata Y. Association between self-reported dental health status and onset of dementia: a 4-year prospective cohort study of older Japanese adults from the Aichi Gerontological evaluation study (AGES) project. Psychosom Med. 2012:74:241-8.

9. Batty GD, Li Q, Huxley R, Zoungas S, Taylor BA, Neal B, et al. Oral disease in relation to future risk of dementia and cognitive decline: prospective cohort study based on the action in diabetes and vascular disease: Preterax and Diamicron modified-release controlled evaluation (ADVANCE) trial. Eur Psychiatry. 2013;28:49-52.

10. Reyes-Ortiz CA, Luque JS, Eriksson CK, Soto L. Self-reported tooth loss and cognitive function: data from the Hispanic established populations for epidemiologic studies of the elderly (Hispanic EPESE). Colombia Medica. 2013;44:139-45.

11. Okamoto N, Morikawa M, Tomioka K, Yanagi M, Amano N, Kurumatani N. Association between tooth loss and the development of mild memory impairment in the elderly: the Fujiwara-kyo study. J Alzheimer's Disease. 2015;44:777-86.

12. Zhu J, Li X, Zhu F, Chen L, Zhang C, McGrath C, et al. Multiple tooth loss is associated with vascular cognitive impairment in subjects with acute ischemic stroke. J Periodont Res. 2015:50:683-8.

13. Takeuchi K, Ohara T, Furuta M, Takeshita T, Shibata Y, Hata J, et al. Tooth loss and risk of dementia in the community: the Hisayama study. J Am Geriatr Soc. 2017;65:e95-e100.
14. Shimazaki Y, Soh I, Saito T, Yamashita Y, Koga T, Miyazaki H, et al. Influence of dentition status on physical disability, mental impairment, and mortality in institutionalized elderly people. J Dent Res. 2001;80:340-5.

15. Hansson P, Eriksson Sörman D, Bergdahl J, Bergdahl M, Nyberg L, Adolfsson $R$, et al. Dental status is unrelated to risk of dementia: a 20-year prospective study. J Am Geriatr Soc. 2014;62:979-81.

16. Naorungroj S, Schoenbach VJ, Wruck L, Mosley TH, Gottesman RF, Alonso A, et al. Tooth loss, periodontal disease, and cognitive decline in the atherosclerosis risk in communities (ARIC) study. Community Dent Oral Epidemiol. 2015;43:47-57.

17. Stewart R, Stenman U, Hakeberg M, Hagglin C, Gustafson D, Skoog I. Associations between oral health and risk of dementia in a 37-year followup study: the prospective population study of women in Gothenburg. J Am Geriatr Soc. 2015;15:100-5.

18. Tsakos G, Watt RG, Rouxel PL, Oliveira C, Demakakos P. Tooth loss associated with physical and cognitive decline in older adults. J Am Geriatr Soc. 2015;63:91-9.

19. Cerutti-Kopplin D, Feine J, Padilha DM, de Souza RF, Ahmadi M, Rompré P, et al. Tooth loss increases the risk of diminished cognitive function: a systematic review and meta-analysis. JDR Clin Trans Res. 2016;1:10-9.

20. Wu B, Fillenbaum GG, Plassman BL, Guo L. Association between oral health and cognitive status: a systematic review. J Am Geriatr Soc 2016;64:739-51.

21. Tonsekar PP, Jiang SS, Yue G. Periodontal disease, tooth loss and dementia: is there a link? A systematic review. Gerodontology. 2017;34:151-63.

22. Imai Y, Nagai K, Sakuma M, Sakuma H, Nakatsuka H, Satoh H, et al. Ambulatory blood pressure of adults in Ohasama. Japan Hypertention. 1993:22:900-12

23. Ohkubo T, Asayama K, Kikuya M, Metoki H, Hoshi H, Hashimoto J, et al. How many times should blood pressure be measured at home for better prediction of stroke risk? Ten-year follow-up results from the Ohasama study. J Hypertens. 2004;22:1099-104.

24. Folstein MF, Folstein SE, McHugh PR. "Mini-mental state". A practica method for grading the cognitive state of patients for the clinician. J Psychiatr Res. 1975;12:189-98.

25. Mori E, Mitani Y, Yamadori A. Usefulness of a Japanese version of the minimental state test in neurological patients (in Japanese). Shinnkei Shinrigaku. 1985:1:2-10.

26. Tsuji I, Imai Y, Nagai K, Ohkubo T, Watanabe N, Minami N, et al. Proposal of reference values for home blood pressure measurement: prognostic criteria based on a prospective observation of the general population in Ohasama. Japan Am J Hypertens. 1997;10:409-18.

27. The Committee of the Japan Diabetes Society on the Diagnostic Criteria of Diabetes Mellitus, Seino Y, Nanjo K, Tajima N, Kadowaki T, Kashiwagi A, et al. Report of the Committee on the Classification and Diagnostic Criteria of Diabetes Mellitus. J Diabetes Investig. 2010;1:212-28.

28. Zung WWK. Depression in the normal aged. Psychosomatics. 1967:8:287-92

29. Williams R. Analyzing Rare Events with Logistic Regression. 2018. https:// www3.nd.edu/ rwilliam/stats3/RareEvents.pdf. Accessed 7 Aug 2018.

30. Aida J, Ando Y, Akhter R, Aoyama H, Masui M, Morita M. Reasons for permanent tooth extractions in Japan. J Epidemiol. 2006;16:214-9.

31. Trovik TA, Klock KS, Haugejorden O. Trends in reasons for tooth extractions in Norway from 1968 to 1998. Acta Odontol Scand. 2000:58:89-96.

32. Gil-Montoya JA, Sanchez-Lara I, Carnero-Pardo C, Fornieles F, Montes J, Vilchez $R$, et al. Is periodontitis a risk factor for cognitive impairment and dementia? A case-control study. J Periodontol. 2015;86:244-53.

33. Shin HS, Shin MS, Ahn YB, Choi BY, Nam JH, Kim HD. Periodontitis is associated with cognitive impairment in elderly Koreans: results from the Yangpyeong cohort study. J Am Geriatr Soc. 2016;64:162-7.

34. Watts A, Crimmins EM, Gatz M. Inflammation as a potential mediator for the association between periodontal disease and Alzheimer's disease. Neuropsychiatr Dis Treat. 2008:4:865-76.

35. Holmes C, Cunningham C, Zotova E, Woolford J, Dean C, Kerr S, et al. Systemic inflammation and disease progression in Alzheimer disease. Neurology. 2009;73:768-74.

36. Sparks Stein P, Steffen MJ, Smith C, Jicha G, Ebersole JL, Abner E, et al. Serum antibodies to periodontal pathogens are a risk factor for Alzheimer's disease. Alzheimers Dement. 2012:8:196-203.

37. Larrieu S, Letenneur L, Helmer C, Dartiques JF, Barberger-Gateau P. Nutritional factors and risk of incident dementia in the PAQUID longitudinal cohort. J Nutr Health Aging. 2004;8:150-4. 
38. Harrison FE. A critical review of vitamin C for the prevention of age-related cognitive decline and Alzheimer's disease. J Alzheimers Dis. 2012;29:711-26.

39. Miller JW, Harvey DJ, Beckett LA, Green R, Farias ST, Reed BR, et al. Vitamin $D$ status and rates of cognitive decline in a multiethnic cohort of older adults. JAMA Neurol. 2015;72:1295-303.

40. Nowjack-Raymer RE, Sheiham A. Numbers of natural teeth, diet, and nutritional status in US adults. J Dent Res. 2007:86:1171-5.

41. Yoshihara A, Watanabe R, Nishimuta M, Hanada N, Miyazaki H. The relationship between dietary intake and the number of teeth in elderly Japanese subjects. Gerodontology. 2005;22:211-8.

42. Momose T, Nishikawa J, Watanabe T, Sasaki Y, Senda M, Kubota K, et al. Effect of mastication on regional cerebral blood flow in humans examined by positron-emission tomography with ${ }^{15} \mathrm{O}$-labelled water and magnetic resonance imaging. Arch Oral Biol. 1997;42:57-61.

43. Onozuka M, Fujita M, Watanabe K, Hirano Y, Niwa M, Nishiyama K, et al. Mapping brain region activity during chewing: a functional magnetic resonance imaging study. J Dent Res. 2002;81:743-6.

44. Kushida S, Kimoto K, Hori N, Toyoda M, Karasawa N, Yamamoto T, et al. Softdiet feeding decreases dopamine release and impairs aversion learning in Alzheimer model rats. Neurosci Lett. 2008;439:208-11.

45. Kato T, Usami T, Noda Y, Hasegawa M, Ueda M, Nabeshima T. The effect of the loss of molar teeth on spatial memory and acetylcholine release from the parietal cortex in aged rats. Behav Brain Res. 1997;83:239-42.

46. Japanese Ministry of Health, Labour and Welfare. The survey of dental disease in 2011. 2012. http://www.mhlw.go.jp/toukei/list/dl/62-23-02.pdf. Accessed 7 Aug 2018. [in Japanese].

47. Naorungroj S, Slade GD, Beck JD, Mosley TH, Gottesman RF, Alonso A, et al. Cognitive decline and oral health in middle-aged adults in the ARIC study. J Dent Res. 2015;92:795-801.

Ready to submit your research? Choose BMC and benefit from:

- fast, convenient online submission

- thorough peer review by experienced researchers in your field

- rapid publication on acceptance

- support for research data, including large and complex data types

- gold Open Access which fosters wider collaboration and increased citations

- maximum visibility for your research: over $100 \mathrm{M}$ website views per year

At $\mathrm{BMC}$, research is always in progress.

Learn more biomedcentral.com/submissions 